\title{
Image Segmentation for Uneven Lighting Images using Adaptive Thresholding and Dynamic Window based on Incremental Window Growing Approach
}

\author{
Rashmi Saini \\ Assistant Professor, G.B.Pant Engg College \\ Pauri Garhwal, 246001, Uttarakhand, India
}

\author{
Maitreyee Dutta, PhD. \\ Associate Professor, National Institute of \\ Technical Teachers Training and Research, \\ (NITTTR) Chandigarh, 160019, India.
}

\begin{abstract}
This paper proposes a novel method to address the problem of segmentation, for uneven lighting images. Though there are many segmentation methods, but most of them are based on either the fixed window method or window merging technique. Limitation of such methods is that, initial size of window is selected manually and segmentation accuracy greatly depends upon the proper choice of initial window size. In the proposed work, problem of uneven illumination condition has been addressed using dynamic window growing approach. The proposed algorithm is based on an incremental window growing approach using entropy based selection criteria. The window thus fixed by the selection criteria are considered as sub-images and each sub-images has been segmented by using minimum standard deviation difference based thresholding to improve the segmentation result. The result of the experiments show that the proposed method can deal with higher number of segmentation problem and improve the overall performance for uneven lighting image segmentation.
\end{abstract}

\section{General Terms}

Thresholding, window size, image binarization, entropy, standard deviation.

\section{INTRODUCTION}

In areas such as image processing and computer vision, image segmentation has been considered a relevant research area due to its wide range of application. Thresholding or image binarization is one of the important technique in image processing and computer vision, to extract the object in an image from the background. The segmented image obtained from thresholding has the advantages of smaller storage space, fast processing speed and ease in manipulation, compared with gray level image which usually contains a large number of gray levels(maximum 256 levels) [3]. Image binarization is central to many applications including document image analysis (printed characters, logos, graphical content), map processing (lines, legends and characters need to be extracted), scene processing, quality inspection of materials, cell images, fingerprint recognition and have a wide range of application in the area of medical image processing[1]. The thresholding techniques can be divided into bilevel and multilevel category. In bilevel thresholding, a threshold is determined to segment the image into two brightness regions, one brightness level correspond to background and another brightness region represent the object within the image. In case of multilevel thresholding, more than one threshold is determined to segment the image into certain brightness regions. Image segmented by using multilevel thresholding techniques represents to one background and several objects of the image. In some cases, however, the existence of some undesired disturbance in thresholding may generate false result. One of the primary disturbance sources that affect the segmentation result is uneven lighting, which often exists in the capturing of an image, especially during field operation. The main causes for uneven lighting are: (a) the light may not be always stable, (b) the object is so large such that it creates an uneven distribution of the light, and (c) the scene is unable to be optically isolated from shadows of other objects [3]. In last two decades a number of methods have been proposed for image segmentation but most of them are not much suitable for uneven lighting images. An ideal adaptive thresholding algorithm would produce the same result when applied to an unevenly light image as a global thresholding algorithm would produce when applied to a perfectly evenly light image. Uneven lighting image segmentation technique can be divided roughly into three categories, pixel wise thresholding, segmentation in partitioned window and window merging techniques. In pixel wise thresholding a threshold is determined for each pixel within the image to be segmented, while in window partitioned method first image is divided into a no of window based on some selection criterion thereafter segment each window individually. This method is known as fixed window method also. In window merging method a selection criterion is tested in each small window, if current window doesn't satisfy the given condition it is merged with the neighbors windows, again selection condition is tested. This procedure is repeated until the given condition satisfied, and then selected window is segmented. One limitation of window merging method is that the segmentation accuracy greatly depends upon the proper choice of initial window size. In this paper we proposed a method to overcome this problem, incremental window growing method using entropy based selection criteria. Selected window is segmented by using minimum standard deviation difference based thresholding Zuoyong Li's Method [12].The paper is organized as follows. Section 2 Introduces the related work, Section 3 discusses the proposed method, section 4 results and analysis, Section 5 is a brief conclusion.

\section{RELATED WORK}

Though there are many segmentation methods most of them are suitable only for even lighting image. Portable digital camera are usually operated under natural illumination, thus result in images with uneven lighting. One possible solution is to divide the whole image into small regions (named windows) and segmenting these windows separately. Theoretically, smaller is the window size, the better is the segmentation result. When the window size becomes too small, it can produce the problem of homogenous windows, i.e., windows contain only background or object pixels, result produce black areas called ghost objects, will occur after thresholding [3]. Meanwhile, if the window size is too large, it 
neither can't treat the uneven lighting condition nor can't get right threshold as well [6]. So there is a need to select window dynamically in order to obtain good segmentation result.

\subsection{Niblack Algorithm}

Niblack's algorithm [10] calculates a pixel-wise threshold by sliding window over the gray level image. The computation of threshold is based on the local mean $\mathrm{m}$ and the standard deviation $\mathrm{s}$ of all the pixels in the window and is given below:

$$
\begin{aligned}
& T_{\text {Niblack }}=m+k \times s \\
& T_{\text {Niblack }}=m+k \sqrt{\frac{\boldsymbol{\Sigma ( \boldsymbol { P } \boldsymbol { i } - \boldsymbol { m } ) ^ { 2 }}}{N P}} \\
& T_{\text {Niblack }}=m+\sqrt{\frac{\Sigma P i^{2}}{N P}-m^{2}}=m+k \sqrt{B}
\end{aligned}
$$

where $N P$ is the number of pixels in the gray image, $\mathrm{m}$ is the average value of the pixels pi, and $\mathrm{k}$ is fixed to -0.2 by the authors. Advantage of Niblack is that it always identifies the text regions correctly as foreground but on the other hand tends to produce a large amount of binarization noise in nontext regions also.

\subsection{Sauvola's Algorithm:}

Sauvola's [8] proposed a method by computing the threshold using the dynamic range of image gray-value standard deviation, $\mathrm{R}$ :

$$
\mathrm{T}_{\text {Sauvola }}=m \times(1-k) \times\left(1-\frac{s}{R}\right)
$$

where $k$ is set to 0.5 and $R$ to 128 . This method outperforms Niblack's algorithm in images where the text pixels have near 0 gray-value and the background pixels have near 255 grayvalues. However, in images where the gray values of text and non-text pixels are close to each other, the results degrade significantly.

\subsection{Zuoyong Li's Method:}

Classic statistical thresholding methods do not consider special characteristic of practical images and fail to obtain satisfying segmentation results [12]. Zuoyong Li's method defines standard deviation difference as criterion for threshold selection, and determines the optimal threshold by minimizing it.

Let I be a gray scale image with $\mathrm{L}$ gray levels $[0,1, \ldots$, $L-1]$. The number of pixels at level $i$ is denoted by $n_{i}$ and the total number of pixels by $N=n_{0}+n_{1}+\ldots+n_{L-1}$. Suppose that the pixels in the image are dichotomized into two classes $C_{l}$ and $C_{2}$ by a gray level $t . C_{1}$ is the set of pixels with levels $[0,1, \ldots, t]$, and $C_{2}$ is the set of pixels with levels $[t+1, \ldots$, $L-1] . C_{1}$ corresponds to the foreground and $C_{2}$ to the background, or vice versa. The mean of each class is given by

$$
\begin{aligned}
& \mu 1=\frac{1}{N 1} \sum_{i=0}^{t} i \times N i, \\
& \mu 2=\frac{1}{N 2} \sum_{i=t+1}^{L-1} i \times N i,
\end{aligned}
$$

where $N_{I}$ and $N_{2}$ are the number of pixels in $C_{l}$ and $C_{2}$, respectively. Furthermore, their respective standard deviation can be formulated as

$$
\begin{aligned}
& \sigma 1=\left(\frac{1}{N 1-1} \sum_{i=0}^{t}(i-\mu 1)^{2} \cdot n i\right)^{1 / 2} \\
& \sigma 2=\left(\frac{1}{N 2-1} \sum_{i=t+1}^{L-1}(i-\mu 2)^{2} \cdot n i\right)^{1 / 2}
\end{aligned}
$$

The new statistical criterion based on standard deviation is defined as

$$
J(t)=\operatorname{abs}(\sigma 1(t)-\sigma 2(t)),
$$

where $a b s$ denotes a common absolute value function.

\section{PROPOSED METHOD}

Image segmentation can be achieved by a number of ways. In the proposed method, we want to overcome the problems undefined initial size of the window. Therefore, we proposed a method in which size of window is determined dynamically based on some parameters of input images. The proposed work is based on two steps: a method to partition the image into dynamic windows by using incremental windowing approach followed by a thresholding technique on those windows. In the proposed work, problem of uneven illumination condition has been addressed using adaptive windowing technique, incremental window growing method using entropy based selection criterion. In the proposed approach, size of selected window is increase by a small incremental value $\Delta \mathrm{w}$ then stopping criteria is tested. This procedure is repeated until the above said condition is satisfied, next window is selected in raster scan manner. The advantages of the proposed approach is that the image is partitioned into a number of windows with different size which contain object as well as background thus reduced the problem of ghost object which appear due to the homogeneous window. Entropy of gray level image is defined as

$$
H=-\sum_{i=1}^{256} P i . \ln P i
$$

where $p_{i}$ is the probability of the $i^{\text {th }}$ gray value. Analogously, the entropy a window $\mathrm{W}$ is defined as

$$
H w=-\sum_{i=1}^{256} P w i . \ln (P w i)
$$

where $p_{w i}$ is the probability of the $i^{\text {th }}$ gray value over the window. A window is selected for segmentation if the following condition is satisfied. If it fails then it is merged with the neighboring windows to form the next level of windows.

$$
\text { If } H w \geq K H
$$

where $K$ is a positive real constant and value of $K$ varies from $0-1$. The notion of fixing $K$ between $0-1$ is to intuitively expect that the maximum entropy of the window could be the entropy of the whole image. 


\section{FLOW CHART FOR PROPOSED ALGORITHM}

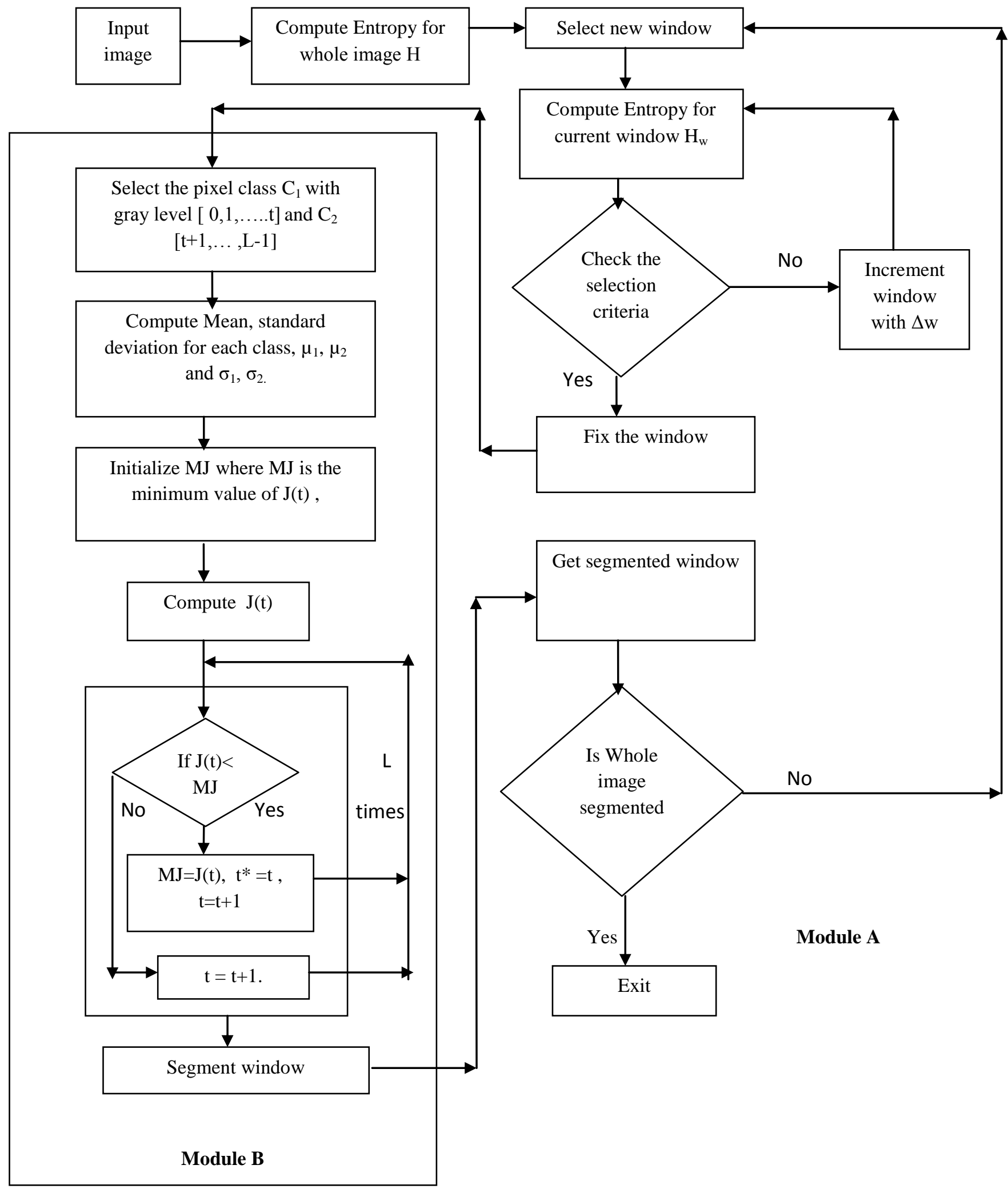

Fig. 1.1: Flow chart for the proposed method 
MODULE A (Zuoyong Li et al. Method):

Define the two categories $C_{1}$ and $C_{2}, C_{1}$ belongs to the background and $C_{2}$ belongs to the object family, or vice-versa. Step 1: Classify each pixel of the input image into $C_{1}$, or $C_{2}$.

Step 2: Compute the mean of both the category $\mu_{1}, \mu_{2}$ using (1) and (2) respectively.

Step 3: Determine standard deviation of both the category $\sigma 1$, $\sigma 2$ using (3) and (4) respectively.

Step 4: Find the statistical criteria using (5). Take the absolute of the difference to generalize the term.

Step 5: Initialize $M j$ a very high value, and $t=0, M j$ represents the minimum of $J(t)$.

Step 6: Compare $J(t)$ and $M j$.If $J(t)<M j$, then $M j=J(t), \quad T h=t$ and increment $t$ by one.

Else only increment $t$ by one.

Step 7: Repeat the previous step $L$ times. Where $L$ is total number of gray level of the image.

Step 8: Segment the window by updated threshold value $T h$.

\section{PROPOSED ALGORITHM:}

\section{MODULE B:}

Step 1: Compute entropy of the whole image using (6).

Step 2: Compute entropy of the selected window using (7).

Step 3: Check out the selection criteria (8).

Step 4: If condition meets then fix the window size and segment the window by module A.

Else increment the size of window by incremental value $\Delta w$ and repeat the procedure from step 2 to step 4 .

Step 5: Stop if whole image is segment.

\section{RESULTS AND ANALYSIS}

We have considered three images with non-uniform illumination condition having different size $(\operatorname{check}(480 \times 680), \operatorname{bird}(256 \times 256), \operatorname{crow}(320 \times 480))$ that are shown in Fig.3(a), 4(a) and 5(a) respectively. The corresponding ground truth image has been generated manually and is shown in Fig.6. All the experiments are performed in MATLAB 7.0 using Core 2 Duo processor $(2.2 \mathrm{GHz})$. Result obtained by using fixed window approach and proposed approach (with varying window size) is shown in fig. 3(c) to fig. 5(c) and fig. 3(d) to fig 5(d) respectively. In order to show the performance of proposed method, in fixed window (size is approximately average of minimum and maximum of window size of the proposed method) method threshold is calculated by using same algorithm that is used to compute threshold value in the proposed method. It can be visualized from segmentation result that the proposed approach gives better performance than the fixed window approach. $M E$ is computed for three images shown in table 1. $M E$ for fixed window method and proposed method (check image) is 0.3286 and 0.2081, (bird image) 0.4451 and 0.0950 , (crow image) 0.3913 and 0.1165 respectively. After analysis we conclude that $M E$ is reduced for each image by the proposed method as compared to fixed window method. Graph shown in fig. 5 (a), (b) and(c) for all three image check, bird and crow respectively, $\mathrm{x}$ axis represent window number, y axis represent the corresponding threshold value for a window. Other parameter considered for result analysis is Computation time, correlation (computed with reference to ground truth images) computed value of these parameters is shown in Table 1.

\section{CONCLUSION}

In this paper, a method is proposed for dynamic size window by using incremental window growing approach to solve the problem of uneven lighting images. As the size of initial window effect the segmentation result, therefore the proposed algorithm is based on the concept of incremental window growing approach, that partition the image into a number of windows with different size. To show the performance of the proposed method, in fixed window method threshold value is computed with using (Module A), the same method as we used for the proposed method. $M E$ computed (Table 1) for the proposed method is reduced as compared to the fixed size window method for all three images. Another advantage of the proposed method is that its segmentation accuracy does not depend on initial window size selection, as this is the one of the major problem of window merging approach. Fig. 2-4 shows the segmentation result with global thresholding method, fixed size window method and proposed method. Experimental result with a variety of real world images demonstrates the effectiveness of the proposed method.

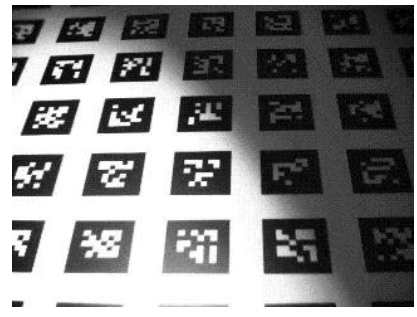

(a) Original Image

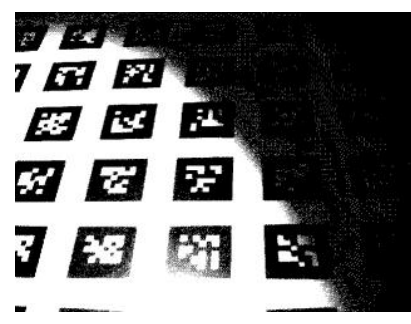

(b) Global Threshold

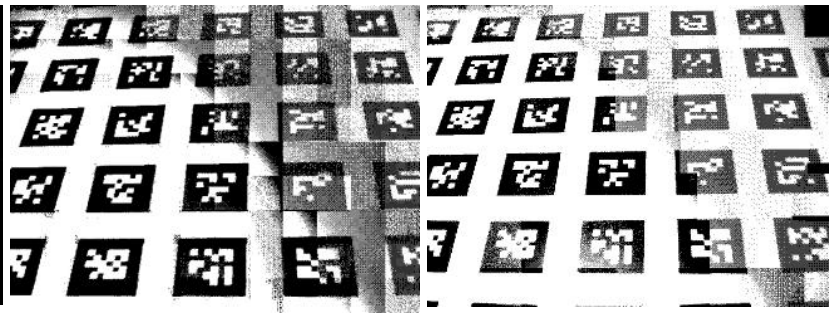

(c) Fix Window (d) Proposed Approach

Fig. 2: (a) original image of check, (b) segmented Image by global threshold, (c) Segmented image by fix sized window and (d) segmented image by varying sized window (proposed approach) 


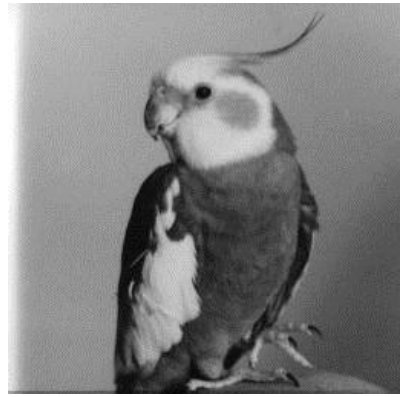

(a) Original Image

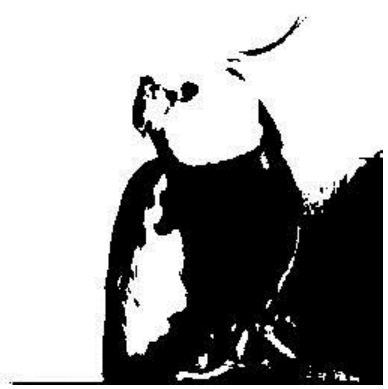

(b) Global Threshold

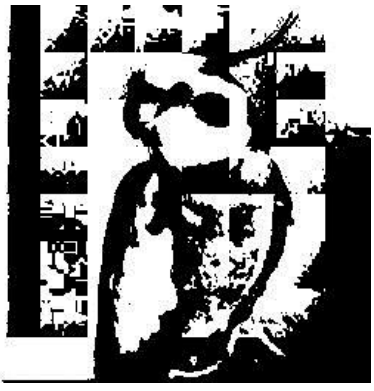

(c) Fix Window

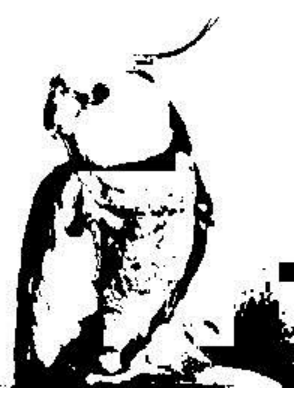

(d) Proposed Approach

Fig. 3: original image of bird, (b) segmented Image by global threshold, (c) Segmented image by fix sized window and (d) segmented image by varying sized window (proposed approach)

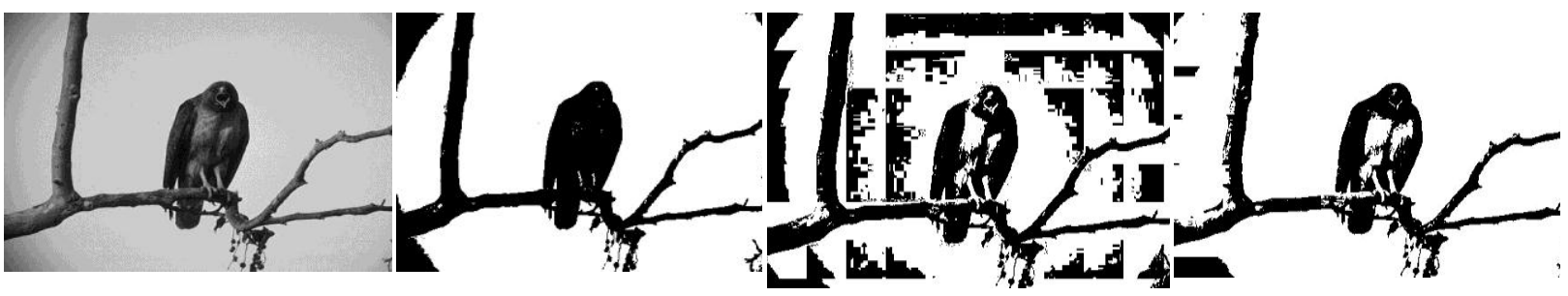

(a) Original Image

(b) Global Threshold

(c) Fix Window

(d) Proposed Approach

Fig. 4: (a) original image of crow, (b) segmented Image by global threshold, (c) Segmented image by fix sized window and (d) segmented image by varying sized window (proposed approach)

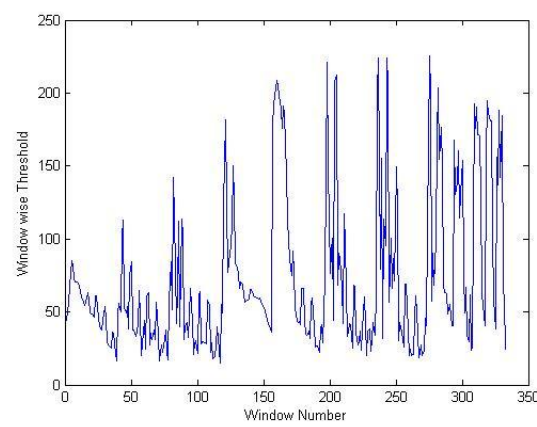

(a) Check

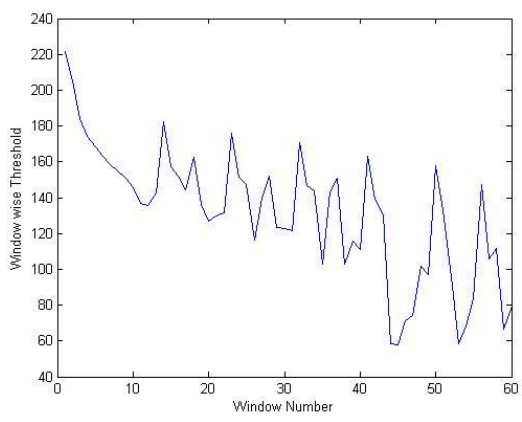

(b) Bird

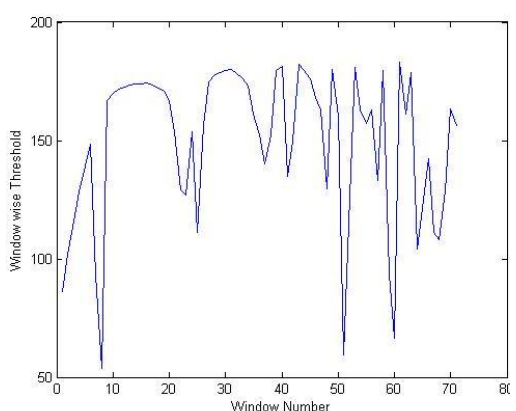

(c) Crow

Fig. 5: (a) Graph showing threshold value of apiece window of Check Image, (b) Graph showing threshold value of apiece window of bird Image and (c) Graph showing threshold value of apiece window of Crow Image

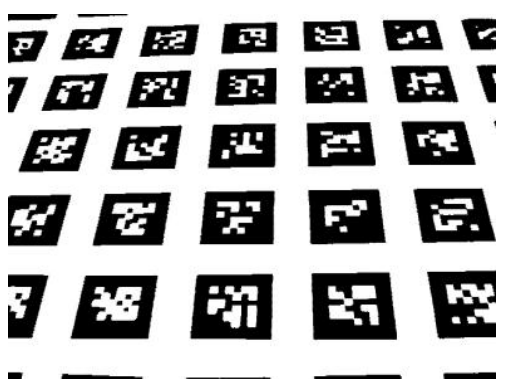

(a) Check

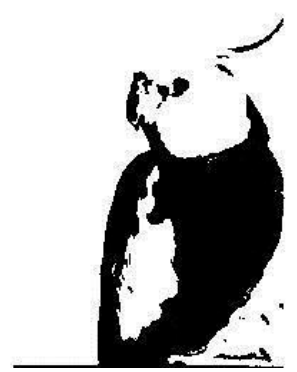

(b) Bird

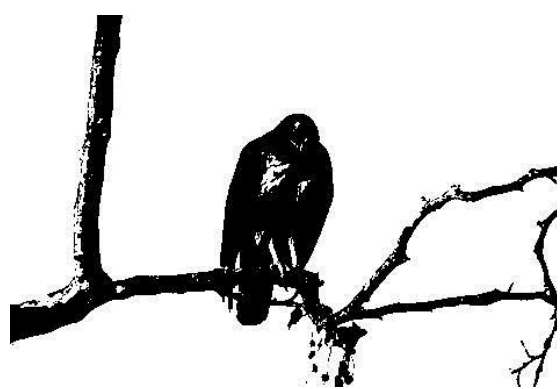

(C) Crow

Fig. 6: (a)Ground truth image of check, (b) Ground Truth image of Bird and (c) Ground Truth image of Crow 
Table 1: Misclassification Error $(M E)$, Correlation and running time for three images.

\begin{tabular}{|c|c|c|c|c|c|c|}
\hline $\begin{array}{l}\text { Image } \\
\text { name } \\
\text { and siz }\end{array}$ & $\begin{array}{l}\text { Window } \\
\text { Size } \\
\text { Minimum, } \\
\text { Maximum }\end{array}$ & $\begin{array}{c}\text { Total } \\
\text { Window }\end{array}$ & $\begin{array}{c}M E \text { with } \\
\text { reference to } \\
\text { ground truth } \\
\text { image and fix } \\
\text { window } \\
\text { method(size) }\end{array}$ & $\begin{array}{c}M E \text { with } \\
\text { reference to } \\
\text { ground } \\
\text { truth image } \\
\text { and } \\
\text { proposed } \\
\text { method }\end{array}$ & $\begin{array}{l}\text { Correlation } \\
\text { between } \\
\text { Ground } \\
\text { truth image } \\
\text { and our } \\
\text { result }\end{array}$ & $\begin{array}{l}\text { Running } \\
\text { time (in } \\
\text { Second) }\end{array}$ \\
\hline $\begin{array}{c}\text { Check } \\
480 \times 640\end{array}$ & 16,79 & 333 & $0.3286(50)$ & 0.2081 & 0.7238 & 8.6130 \\
\hline $\begin{array}{c}\text { Bird } \\
256 \times 256\end{array}$ & 8,45 & 60 & $0.4451(30)$ & 0.0950 & 0.5940 & 1.9375 \\
\hline $\begin{array}{c}\text { Crow } \\
320 \times 480\end{array}$ & 12,54 & 71 & $0.3913(44)$ & 0.1165 & 0.7487 & 3.9063 \\
\hline
\end{tabular}

\section{REFERENCES}

[1] Naveed Bin Rais, M. Shehzad Hanif and rmtiaz A. Taj” Adaptive Thresholding Technique for Document Image Analysis"INMIC $8^{\text {th }}$ International Conference IEEE pp.61-66, 2004.

[2] P. Kanungo P. K. Nanda A. Ghosh" Parallel Genetic Algorithm based adaptive thresholding for image segmentation under uneven lighting conditions" IEEE International Conference on System Man and cybernetics, pp. 1904-1911, 2010.

[3] Q. Huang, W. Gao, W. Cai, "Thresholding technique with adaptive window selection for uneven lighting image," Pattern Recognition Letters, vol. 26, pp. 801808, 2005.

[4] S.Farid, F.Ahmed" Application of Niblack's Method on Images" IEEE International Conference on Emerging Technologies, 2009.

[5] Mehmet Sezgin, B. Sankur" Survey over image thresholding techniques and quantitative performance evaluation" Journal of Electronic Imaging Vol. 13(1), pp146-165, 2004.

[6] Guoqing Gu Wenwen Han" Adaptive window based Uneven Lighting Document Segmentation IEEE Ninth International conference on Document Ananlysis and Recognition .pp.713-716, 2007.

[7] Zuoyong Lia, Chuancai Liu, Guanghai Liu, Yong Cheng, Xibei Yang, Cairong Zhao," A novel statistical image thresholding method" Int. J. Electron. Commun. 64, 1137-1147, 2010.

[8] J. Sauvola, M. Pietikainen, "Adaptive Document Image Binariation", Pattern Recognition, vol. 33, pp. 225-236, 2000.

[9] Graham Leedham, Chen Yan, Kalyan Takru, Joie Hadi Nata Tan and Li Mian "Comparison of Some Thresholding Algorithms for Text/Background Segmentation in Difficult Document Images"IEEE International conference on Document Ananlysis and Recognition(ICDAR) pp.859-864, 2003.
[10] W. Niblack, An Introduction to Digital Image Processing. Prentice Hall, Englewood Cliffs, 1986.

[11] Khurram Khurshid, Imran Siddiqi, Claudie Faure, Nicole Vincent " Comparison of Niblack inspired Binarization methods for ancientdocuments",International Conference on Knowledge Discovery and Retrival(KDIR) pp.198-193, 2010.

[12] Zuoyong Li, Yong Cheng, Chuancai Liu, Cairong Zhao, "Minimum Standard Deviation Difference-Based Thresholding" IEEE International Conference on Measuring Technology and Mechatronics Automation(ICMTMA), pp. 664-667, 2010.

[13] Satyabrat Srikumar, Mamta Wagh, P.K. Nanda, "Adaptive Windowing and Granular Computing based Image Segmentation" IEEE International Conference on Energy Automation and Signal(ICEAS), pp. 1-5, 2011.

[14] Graham Leedham, Chen Yan, Kalyan Takru, Joie Hadi Nata Tan and Li Mian, "Comparison of Some Thresholding Algorithms for Text/Background Segmentation in Difficult Document Images" IEEE International conference on Document Ananlysis and Recognition (ICDAR) pp.859-864, 2003.

[15] Jaskirat Kaur, Sunil Agrawal and Renu Vig. "A Comparative Analysis of Thresholding and Edge Detection Segmentation Techniques". International Journal of Computer Applications 39(15):29-34, February 2012.

[16] Ch.v. Narayana, Sreenivasa E Reddy and Seetharama M Prasad. "Automatic Image Segmentation using Ultra Fuzziness" International Journal of Computer Applications 49(12):6-13, July 2012.

[17] Sayantan Nath, Dr. Sonali Agarwal and Qasima Abbas Kazmi. "Image Histogram Segmentation by Multi-Level Thresholding using Hill Climbing Algorithm" International Journal of Computer Applications 35(1):63-72, December 2011 